Supporting Information for:

\title{
Role of tethered ion placement on polymerized ionic liquid structure and conductivity: \\ Pendant vs backbone charge placement
}

Christopher M. Evans, Colin R. Bridges, Gabriel E. Sanoja, Joshua Bartels and Rachel A. Segalman

Materials. Azobisisobutyronitrile (AIBN, Aldrich), N-acryloxy succinimide (TCI Chemicals), cyanomethyl dodecyl trithiocarbonate (CMDDT, Aldrich), 3-(amino propyl) imidazole (Aldrich), methyl iodide (Spectrum Chemical), lithium bis(trifluoromethane sulfonimide) (LiTFSI, Aldrich), and triethylamine (TEA, Aldrich) were used as received. Anhydrous dimethyl formamide (DMF, Aldrich) and dimethyl sulfoxide (DMSO, Aldrich) were used as received for polymerizations.

Synthesis. Pendant PIL homopolymers were synthesized as depicted in Scheme 1 starting with synthesis of the poly(n-acryloxy succinimide) (PASI). N-acryloxy succinimide was dissolved in anhydrous DMF $(0.5 \mathrm{~g} / \mathrm{mL})$ followed by addition to a $25 \mathrm{~mL}$ Schlenk flask of CMDDT and AIBN in a 10:1 molar ratio. A minimum of three freeze-pump-thaw cycles were used to remove dissolved oxygen from the system. The reaction was run at $70{ }^{\circ} \mathrm{C}$ for $18 \mathrm{~h}$ followed by precipitation in methanol. The PASI was dissolved in anhydrous DMSO with 1.2 eq. of 3-(amino propyl) imidazole and 1 eq. of TEA and reacted overnight at $60{ }^{\circ} \mathrm{C}$. This polymer was then dissolved in methanol with 1.2 eq. of methyl iodide to quaternize the free nitrogen. Salt metathesis was performed using a 10x molar eq. of LiTFSI in methanol at $50^{\circ} \mathrm{C}$ to exchange $\mathrm{TFSI}^{-}$for $\mathrm{Br}^{-}$and the polymer was dialyzed for $48 \mathrm{~h}$ in DI water and $24 \mathrm{~h}$ in methanol followed by drying at $120^{\circ} \mathrm{C}$ overnight under vacuum. The formation of PILs was confirmed using ${ }^{1} \mathrm{H}$ NMR on a 600 MHz Bruker spectrometer in d-DMSO. The pendant PIL NMR has been previously reported. $^{19}$ 


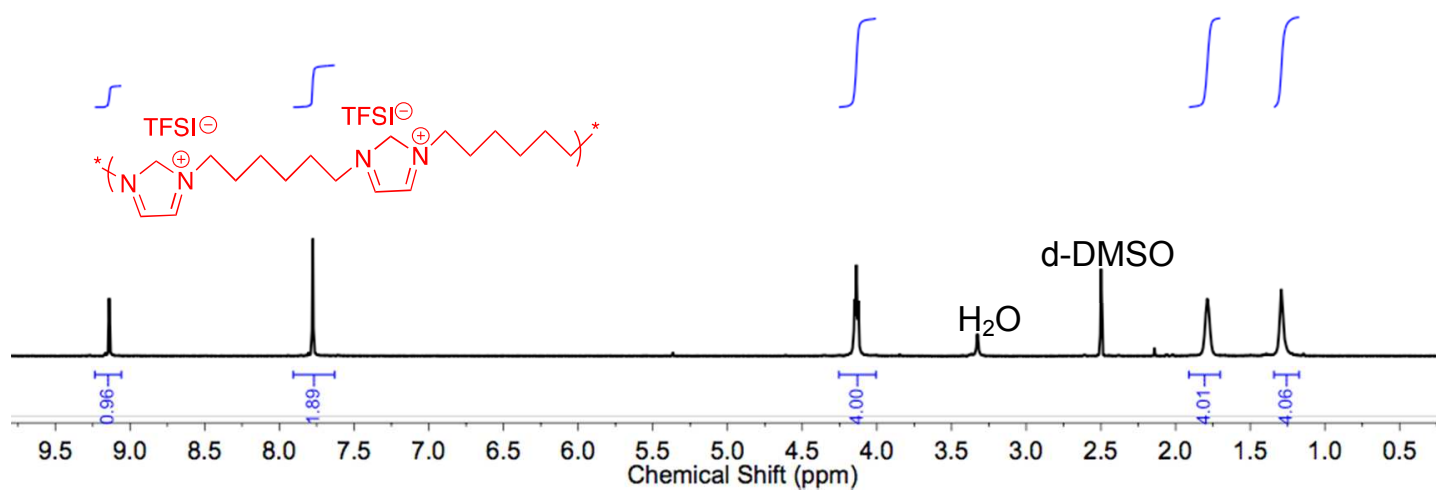

Figure S1. ${ }^{1}$ H NMR spectra in d-DMSO confirms the formation of backbone PIL. Three distinct groups of four methylene protons are observed between imidazolium groups as anticipated.

To determine relative molecular weights, dimethyl formamide GPC was performed with

$0.25 \% \mathrm{LiBr}$ added to screen the charges. Although the MW determination was against PS

standards, we note that the solution properties and interaction with the column should be similar between the backbone and pendant PILs such that they can be reliably compared. The close agreement in $\mathrm{M}_{\mathrm{n}}$ of $22.1 \mathrm{~kg} / \mathrm{mol}$ (backbone) and $24.2 \mathrm{~kg} / \mathrm{mol}$ (pendant) indicates that the molecular weights are not substantially different and should not play a major role in the conductivity and structural trends.

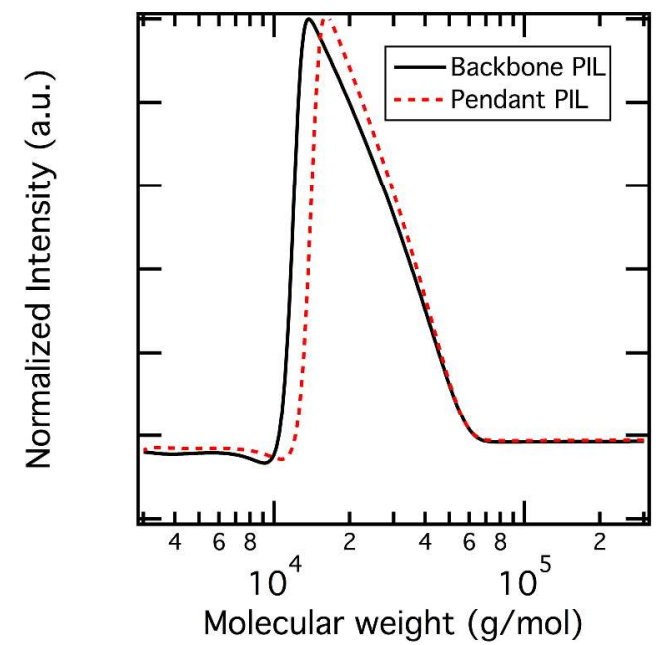

Figure S2. Molecular weight distributions for the backbone (black solid line) and the pendant (red dashed line) PILs. GPC was run in a DMF column with $0.25 \% \mathrm{LiBr}$ added to screen charges. The lower MW portion is truncated due to dialysis of the materials. 
Conductivity measurements. Impedance measurements were performed on a Biologic VSP-300 using two circular stainless steel electrodes with Kapton tape as an $\sim 50$ micron spacer. A hole of precise diameter was punched in the tape to control the electroactive area of the sample. A second electrode was placed on top and then consolidated with a Carver press $\left(140^{\circ} \mathrm{C}, 1 \mathrm{ton}, 5 \mathrm{~min}\right)$. The film thicknesses were measured using a digital micrometer ( $+/-1 \mu \mathrm{m}$ precision) on the assembly with and without the PIL. Impedance measurements were performed from $3 \mathrm{MHz}$ down to $30 \mathrm{mHz}$ with an amplitude of 100 $\mathrm{mV}$ and the complex impedance $\left(Z^{*}=Z^{\prime}-i \omega Z^{\prime \prime}\right)$ was converted to complex conductivity according to:

$$
\sigma^{\prime}(\omega)=\frac{Z^{\prime}(\omega)}{k\left[Z^{\prime}(\omega)^{2}+Z^{\prime \prime}(\omega)^{2}\right]}
$$

The frequency independent plateau of conductivity was taken as the "DC conductivity". Samples were dried in a vacuum oven overnight at $120^{\circ} \mathrm{C}$ followed by hot pressing and another $2 \mathrm{~h}$ under vacuum. Conductivity measurements were performed on an INSTEC hot stage with a continuous dry nitrogen purge provided by a liquid nitrogen dewar. The samples were equilibrated for $\sim 1 \mathrm{~h}$ at the highest temperature and the conductivity was monitored until it stabilized. Measurements were then taken upon cooling at a rate of $\sim 3$ ${ }^{\circ} \mathrm{C} / \mathrm{min}$. 
The conductivity of the backbone PIL is higher than that of the pendant even after plotting on a $\mathrm{T}-\mathrm{T}_{\mathrm{g}}$ axis. Additionally, a $\mathrm{T} / \mathrm{T}_{\mathrm{g}}$ axis produces the same results as shown in

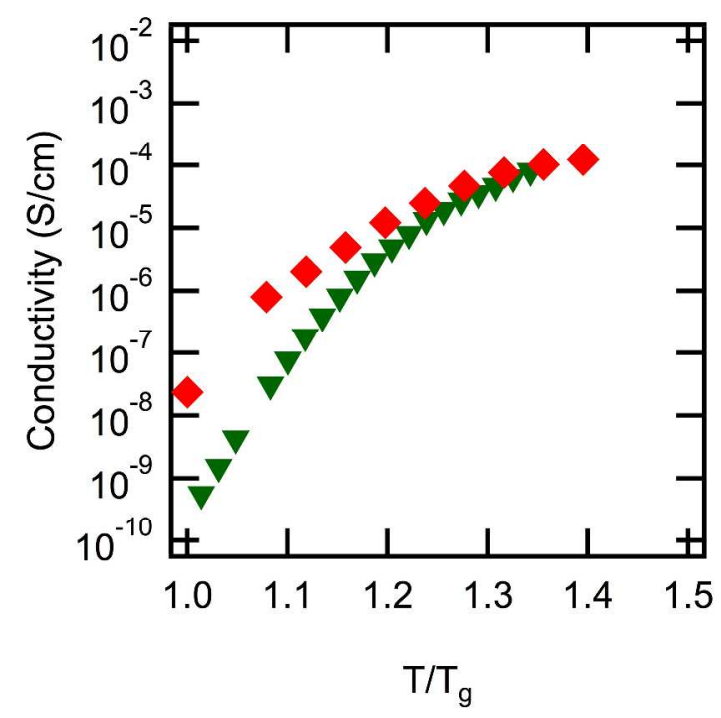

Figure S3. Backbone PIL (red diamonds) and pendant PIL (green triangles) conductivity on a $\mathrm{T} / \mathrm{T}_{\mathrm{g}}$ plot produces the same qualitative trends as the $\mathrm{T}-\mathrm{T}_{\mathrm{g}}$ plot.

$\mathbf{X}$-ray scattering. Samples for small and wide-angle xray scattering were prepared by removing the polymer from the conductivity cells and placing the material in a washer with Kapton windows attached via silicone epoxy to provide a moisture barrier. The assembly was formed in a nitrogen glove box and samples were stored over Drierite until they were placed in the beamline. Experiments were performed at both beamline 7.3.3 at the Advanced Light Source at Lawrence Berkeley National Lab and on a benchtop xray scattering setup in the MRL at UCSB. Data analysis was performed using the Nika package for Igor pro and sample to detector distances were calibrated with AgB. Sample intensities were background corrected by subtracting the signal of an empty sample holder, with both sample and blank intensities normalized by their total transmission. 


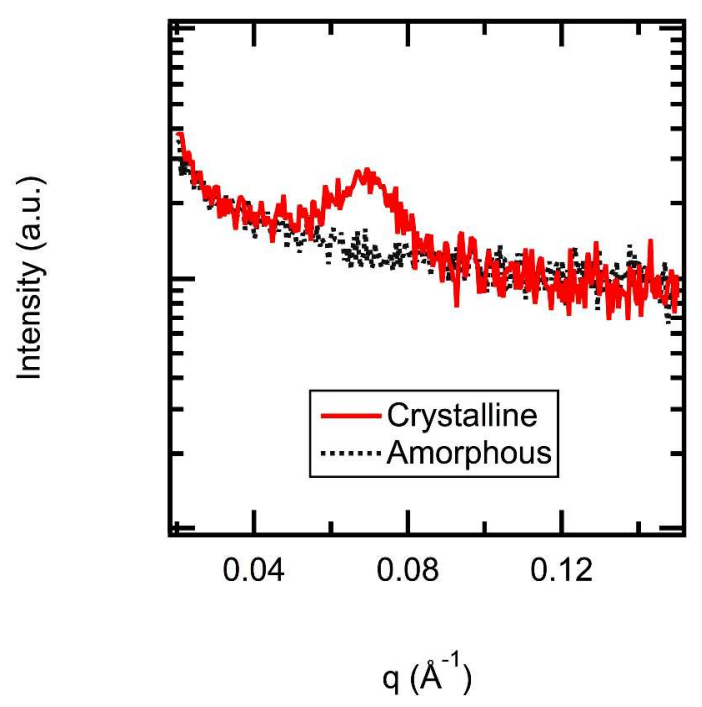

Figure S4. SAXS of the backbone PIL in the crystalline state (red line) and the amorphous state after freshly melting the sample (dotted black line). The "ionic aggregate" peak is only observed in the crystalline form of the material. Data were taken on a benchtop SAXS hench the worse signal to noise compared to the synchrotron data of Figure 2.

To gain further insights into the backbone PIL melting and crystallization, DSC experiments were performed on a Perkin-Elmer DSC 8000 calibrated with indium and zinc for temperature and indium for heat flow. StepScan experiments were performed with $0.5^{\circ} \mathrm{C}$ steps and a heating rate of $5{ }^{\circ} \mathrm{C} / \mathrm{min}$ between points. All samples were taken from a master batch of crystallized material and a new piece was used for each experiment such that the thermal history is identical. 


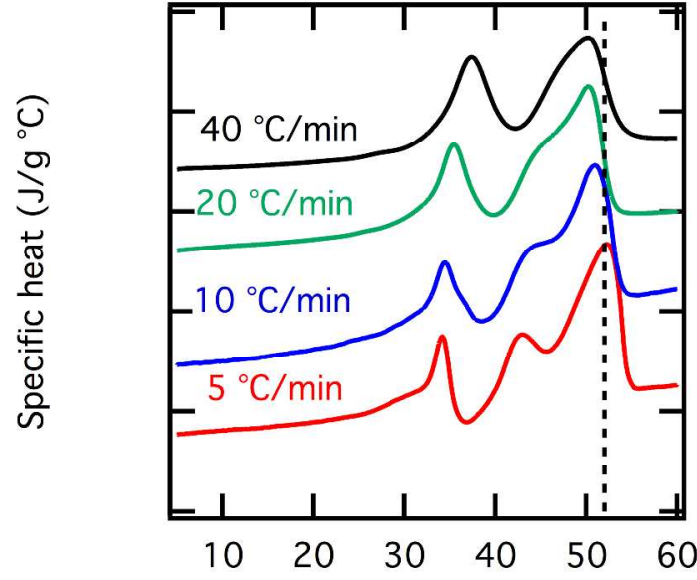

Temperature $\left({ }^{\circ} \mathrm{C}\right)$

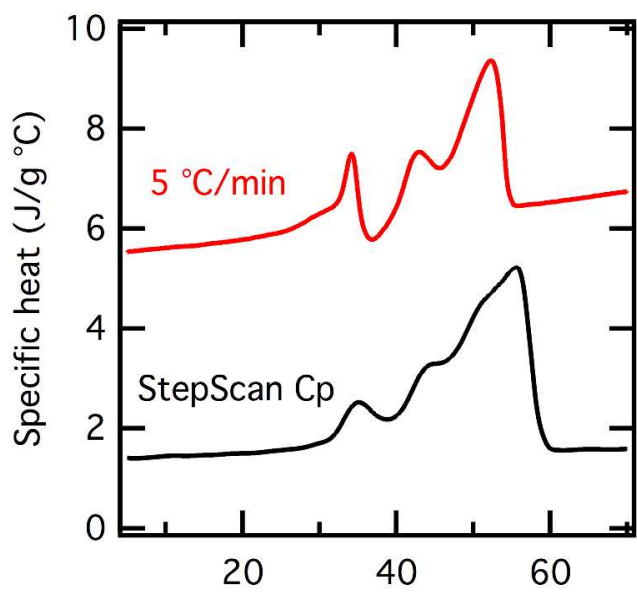

Temperature $\left({ }^{\circ} \mathrm{C}\right)$

Figure S5. a) Variable scan rate DSC experiments for the ionene crystallized at $20^{\circ} \mathrm{C}$ overnight. The two highest endotherms merge with increasing scan rate consistent with melt-recrystallization. At the slowest rate $\left(5^{\circ} \mathrm{C} / \mathrm{min}\right)$, a low temperature shoulder is observed assigned to the rigid amorphous fraction followed by a melting peak assign to secondary crystal melting. A dip corresponding to cold crystallization is also observed which is absent in the reversible heat flow curve obtained via StepScan DSC measurements. Ticks on the ordinate of a) correspond to $2 \mathrm{~J} / \mathrm{g}{ }^{\circ} \mathrm{C}$ and have been shifted vertically for clarity. The StepScan data is on an absolute heat capacity scale. 